\title{
A novel anatomical classification of the frontal sinus: can it be useful in clinical approach to frontal sinusitis?
}

\author{
Meltem Özdemir ${ }^{1 *}$ (D, Rasime P. Kavak ${ }^{1}$ (D) Bülent Öcal ${ }^{2}$ (D) and Handan Soysal ${ }^{3}$ (D)
}

\begin{abstract}
Background: We aimed to test the clinical relevance of a newly introduced anatomical classification system of the frontal sinus and to investigate the relation between the frontal sinus type according to this system and the development of frontal sinusitis. We retrospectively evaluated the computed tomography images of 808 frontal sinuses of 404 patients and classified the sinuses as small, medium-sized, and large, based on their size and relation to the orbital roof. We related this classification to the presence or absence of the findings of frontal sinusitis including mucosal thickening, retention cyst/polyp, and/or fluid collection.

Results: We found that the most common frontal sinus type is medium-sized (65.84\%), followed by the small (22.89\%) and large (11.26\%) types, respectively. There was no significant difference between the right and left sides in terms of frontal sinus type $(P<0.05)$. We recorded sinusitis in $28(15.1 \%)$ small, 180 (33.8\%) medium-sized, and 40 (43.9\%) large sinuses. And we showed that the prevalence of sinusitis in medium-sized and large sinuses is significantly higher than that in small sinuses for both sides ( $P$ values were 0.001 and 0.015 , respectively for the right, and 0.005 and 0.001 , respectively for the left side).

Conclusion: The result obtained in this study may be considered the first step in demonstrating the clinical benefit of this classification. However, there is no doubt that further comprehensive studies with large series are needed to fully determine the clinical relevance of this newly introduced classification system.
\end{abstract}

Keywords: Frontal sinus, Classification, Frontal sinusitis, CT

\section{Background}

Frontal sinuses are a pair of pneumatic cavities whose development begins in the fourth month of fetal life and continues to grow until the age of 20 years. The two sinus cavities are separated by a septum, are almost always asymmetrical, and both extend back into the orbital portion of the frontal bone [1]. Frontal sinuses are known to be one of the most variable anatomic structures of the human body. There exists a great range of variation in terms of size, shape, and drainage pathways among frontal sinuses of different individuals [2].

\footnotetext{
* Correspondence: meltemkaan99@gmail.com

'Department of Radiology, University of Health Sciences, Dışkapı Yıldııım

Beyazıt Training and Research Hospital, Ankara, Turkey

Full list of author information is available at the end of the article
}

Anatomical variations of the frontal sinuses are shown to be closely related to the physiopathology, symptomatology, clinical presentation, development of complications, and the treatment of frontal sinusitis [3-8]. However, a widely accepted anatomical classification of the frontal sinuses has not yet been developed. In the previous studies on the anatomic variations, the authors used the terms such as aplasia, hypoplasia, medium-size, and hyperplasia to define the volumetric variants of the frontal sinus $[9,10]$. Or they tried to relate the development of pathologies to the presence or absence of special variants such as frontal recess cells [6-8]. The classification system described by Bent and Kuhn [11] as well as the newly described IFAC [12] has been criticized for many aspects of clinical usability $[13,14]$.

\section{Springer Open}

(๑) The Author(s). 2021 Open Access This article is licensed under a Creative Commons Attribution 4.0 International License, which permits use, sharing, adaptation, distribution and reproduction in any medium or format, as long as you give appropriate credit to the original author(s) and the source, provide a link to the Creative Commons licence, and indicate if changes were made. The images or other third party material in this article are included in the article's Creative Commons licence, unless indicated otherwise in a credit line to the material. If material is not included in the article's Creative Commons licence and your intended use is not permitted by statutory regulation or exceeds the permitted use, you will need to obtain permission directly from the copyright holder. To view a copy of this licence, visit http://creativecommons.org/licenses/by/4.0/. 
In their very recent study, Stokovic et al. showed that there is a significant relationship between the measures of the frontal sinus size parameters obtained on the coronal plane and the extent of the frontal sinus-orbital roof contact area. Furthermore, using cluster analysis, they identified three types of frontal sinus based on size and relation to the orbital cavity [15]. This is a very simple classification that gives the opportunity to identify the frontal sinus type easily on the conventional sinus radiograph and promises to be very useful in clinical practice if it is determined to be significantly related to the development of pathological processes. The aim of the current study was to investigate the relation between the development of frontal sinusitis and the frontal sinus type according to the classification proposed by Stokovic et al. [15].

\section{Methods}

\section{Patient population and study design}

With institutional review board approval, radiological records of a total of 404 patients who underwent CT of the paranasal sinuses between January 2018 and January 2019 because of headache, nasal obstruction, anosmia, facial pain, or facial trauma were retrospectively evaluated. There were 215 men and 189 women with a mean age of 39.8 years (range 18 to 88). Approval was provided by [Ethics Committee of University of Health Sciences, Dışkapı Yıldırım Beyazıt Training and Research Hospital].

\section{CT protocol and image analysis}

Imaging was performed using a 128-slice CT scanner (Optima CT 660, GE Healthcare System, Milwaukee, USA) using the following parameters: $120 \mathrm{kV}$; $150 \mathrm{mAs}$; slice thickness $=0.5 \mathrm{~mm} ; \mathrm{FOV}=18-24 \mathrm{~cm}$. The left and right sinuses were evaluated and recorded separately.

As proposed by Stokovic et al. [11], we classified the frontal sinuses as small, medium-sized, and large according to the extent of pneumatization of the orbital roof in the coronal plane. The coronal section in which the frontal sinus is depicted in its widest diameter was chosen to classify the sinus. For a practical purpose, we divided the orbital roof into three equal parts and made the classification based on this division (Fig. 1):

1. Small: orbital roof pneumatization is absent or only the medial third of the roof is pneumatized (Fig. 2a).

2. Medium-sized: pneumatization of the medial third and a portion of the central third of the orbital roof (Fig. 2b).

3. Large: pneumatization involving the medial, central, and a portion or all of the lateral third of the orbital roof (Fig. 2c).

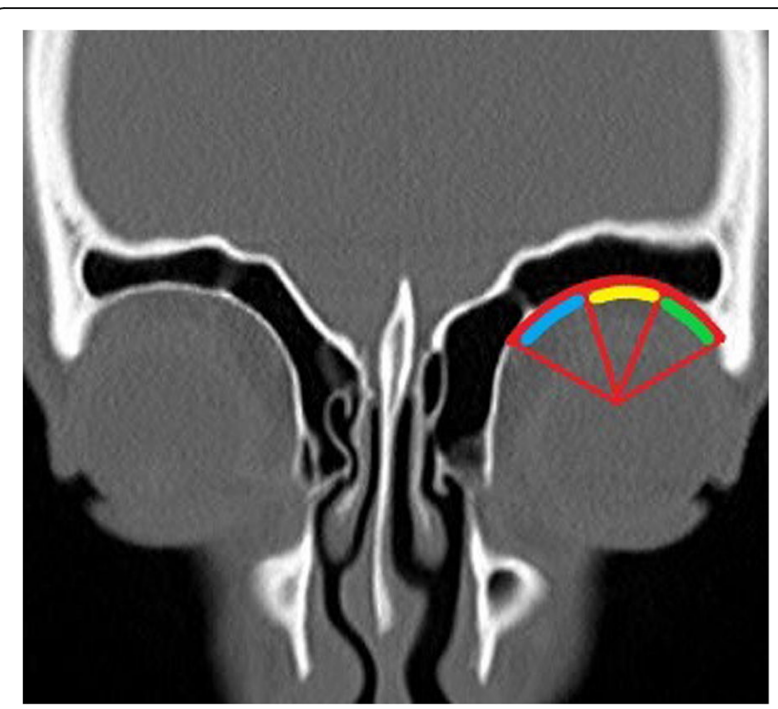

Fig. 1 Coronal computed tomography section demonstrating the division of the orbital roof in three equal parts. Medial, central, and lateral thirds of the roof are shown by blue, yellow, and green lines, respectively

The cases showing at least one of the following pathological findings in at least one of the coronal, axial, or sagittal frontal sinus CT sections were accepted as frontal sinusitis:

1. Mucosal thickening: Mucosal thickening of $>3 \mathrm{~mm}$ was accepted as a positive finding (Fig. 3a).

2. Retention cyst and polyp: As retention cysts and polyps cannot be exactly differentiated on CT, they were joined in the same group. A hemispherical or dome-shaped, well-circumscribed, homogeneous area with a smooth outline within the sinus was accepted as a positive finding (Fig. 3b).

3. Fluid collection: An air-fluid level within the sinus was accepted as a positive finding (Fig. 3c).

Two experienced radiologists reviewed the frontal sinuses independently. In the cases in which a discrepancy occurred in the interpretations of the images, a common re-examination was performed and the final decisions were made by consensus.

\section{Statistical analysis}

The normality of distribution of continuous variables was tested by Shapiro-Wilk test. Mann-Whitney $U$ test was used to compare two independent groups for nonnormal data. Chi-square test was performed to investigate the relationship between categorical variables, and when chi-square test result is significant, Bonferroni correction was applied to adjust $P$ values for multiple comparisons. McNemar-Bowker and McNemar tests were applied to compare categorical and ordinal variables 


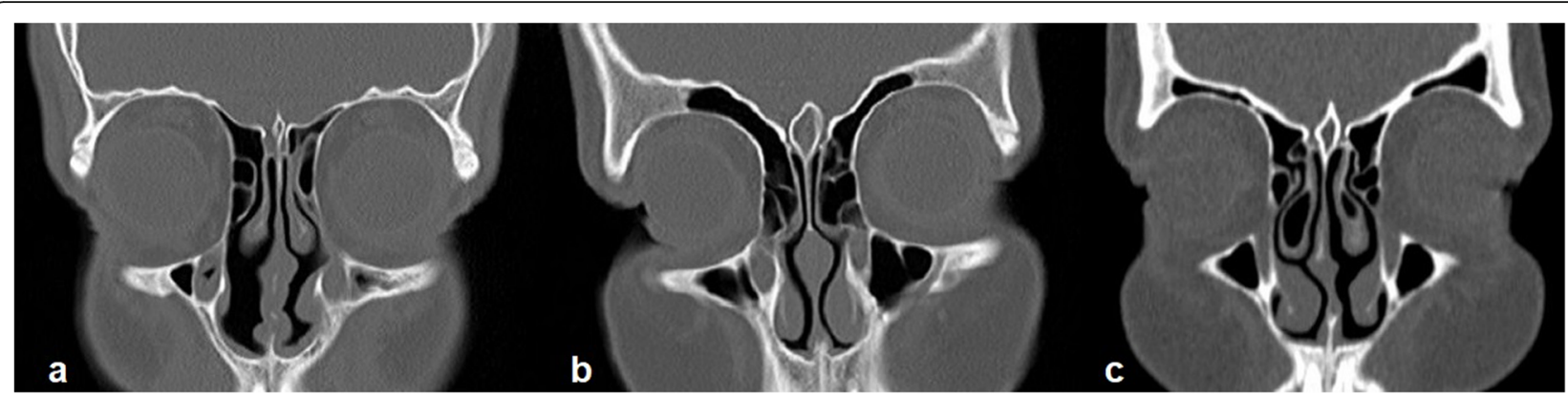

Fig. 2 Coronal computed tomography sections demonstrating the small (a), medium-sized (b), and large (c) types of frontal sinuses according to their relation to the orbital roof

measured from left and right sides of the patients. All statistical analyses were performed with SPSS for Windows version 24.0 and a $P$ value $<0.05$ was accepted as statistically significant.

\section{Results}

As the result of the evaluation of 808 frontal sinuses of 404 patients in the study group, the findings recorded in terms of the frequency of sinus types and sinusitis are summarized in Table 1 . We recorded that the most common frontal sinus type is medium-sized $(65.84 \%)$ followed by small $(22.89 \%)$ and large $(11.26 \%)$ types, respectively. There was no significant difference between the right and left sides in terms of frontal sinus type (Table 2).

We recorded sinusitis in $156(38.6 \%)$ patients. Of 808 frontal sinuses, 248 (30.7\%) showed one or more findings of sinusitis. There was no significant difference between the right and left frontal sinuses in terms of the prevalence of sinusitis $(P=0.982)$ (Table 3$)$. The prevalence of frontal sinusitis did not differ by age $(P=0.151)$. However, we showed that the prevalence of frontal sinusitis among men is significantly higher than that among women $(P=0.008)$ (Table 4$)$.
There was at least one finding of sinusitis in 28 (15.1\%) small, 180 (33.8\%) medium-sized, and 40 (43.9\%) large sinuses. Table 5 demonstrates the relationship between the type of frontal sinus and the frequency of sinusitis for both sides. We recorded a significant relationship between the type of frontal sinus and the frequency of sinusitis for both the right and the left sides. The prevalence of sinusitis in medium-sized and large sinuses was significantly higher than that in small sinuses for both sides ( $P$ values were 0.001 and 0.015 , respectively for the right side, and 0.005 and 0.001 , respectively for the left side). Although we recorded that as the size of the frontal sinus increased, the frequency of sinusitis increased, the difference between the medium-sized and large types in terms of the frequency of sinusitis did not reach the level of statistical significance. No significant difference between the right and left sides was noted in terms of the frequency of sinus types among patients with sinusitis.

\section{Discussion}

Stokovic et al. have carried out a comprehensive study on CT-based measurements of the frontal sinus

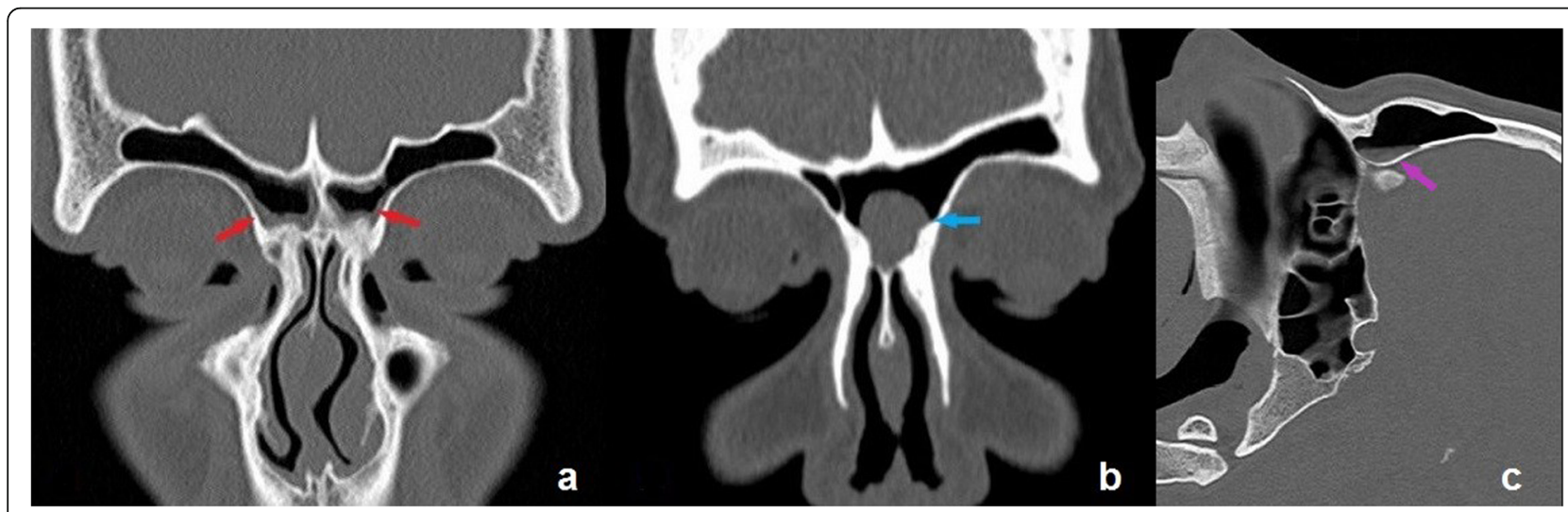

Fig. 3 Coronal (a, b) and sagittal (c) computed tomography sections demonstrating mucosal thickening (red arrows), retention cyst/ polyp (blue arrow), and fluid collection (purple arrow) which are taken as the criteria of the presence of frontal sinusitis 
Table 1 The findings recorded in terms of sinusitis and sinus type in 808 frontal sinuses of 404 patients in the study group

\begin{tabular}{lll}
\hline & N & \% \\
\hline Frontal sinus type & & \\
Right & 97 & 24.0 \\
$\quad$ Small & 268 & 66.3 \\
$\quad$ Medium-sized & 39 & 9.7 \\
$\quad$ Large & & \\
Left & 88 & 21.8 \\
$\quad$ Small & 264 & 65.3 \\
$\quad$ Medium-sized & 52 & 12.9 \\
$\quad$ Large & & \\
Frontal sinusitis findings & 156 & 38.6 \\
Patients with frontal sinusitis & 120 & 29.7 \\
Right frontal sinusitis & 128 & 31.7 \\
Left frontal sinusitis & & \\
\hline
\end{tabular}

dimensions of cadaveric skulls and displayed the extreme variability of the frontal sinuses in terms of size and shape. They showed that the coronal extensions of the frontal sinuses are proportional to their sagittal extent and suggested a highly practical anatomical classification of the frontal sinuses as a candidate for a universally accepted classification system. We aimed to test the clinical relevance of this system and investigated the relationship between the frontal sinus type and the development of frontal sinusitis. We evaluated 808 frontal sinuses of 404 patients in terms of their types according to this novel classification system and the presence or absence of the findings of frontal sinusitis. We found a significant relationship between the type of the frontal sinus and the frequency of frontal sinusitis. The prevalence of sinusitis in medium-sized and large sinuses was significantly higher than that in small sinuses. Although we recorded that as the size of the frontal sinus increased, the frequency of sinusitis increased, the difference between the medium-sized and large types in terms
Table 3 Comparison of the right and left sides in terms of the frequency of frontal sinusitis

\begin{tabular}{|c|c|c|c|c|c|c|}
\hline & \multicolumn{6}{|c|}{ Right frontal sinusitis } \\
\hline & \multicolumn{2}{|c|}{ Positive } & \multicolumn{2}{|c|}{ Negative } & \multicolumn{2}{|c|}{ Total } \\
\hline & $\bar{N}$ & $\%$ & $\bar{N}$ & $\%$ & $\bar{N}$ & $\%$ \\
\hline \multicolumn{7}{|c|}{ Left frontal sinusitis } \\
\hline Positive & 92 & 76.7 & 36 & 12.7 & 128 & 31.7 \\
\hline Negative & 28 & 23.3 & 248 & 87.3 & 276 & 68.3 \\
\hline Total & 120 & 100.0 & 284 & 100.0 & 404 & 100.0 \\
\hline
\end{tabular}

of the frequency of sinusitis did not reach the level of statistical significance.

In line with the results of the previous studies on the size of the frontal sinus, we recorded that the most common type is medium-sized frontal sinus type $[9,10,15]$. And in consistence with the study conducted by Stokovic et al, we found no significant side-related difference in the sizes of the frontal sinuses [15].

Sinusitis is one of the most common diseases being dealt with in primary health-care and mostly diagnosed based on the combination of clinical findings and conventional sinus radiography $[16,17]$. The frontal sinus is the most complex one among all paranasal sinuses. And because of its proximity to the anterior cranial fossa and orbita, frontal sinusitis is one of the two most common sources of orbital and cranial complications with ethmoidal sinusitis, among other sinus infections $[18,19]$. The results of the recent studies strongly suggest that the anatomical variations of frontal sinus in terms of size and shape are closely related to the development of both sinusitis and its complications [3-8]. There are studies reporting a significant increase in the frequency of frontal sinusitis in the presence of certain types of frontal recess cells [6-8]. Tezer et al. recorded a significantly higher frequency of frontal sinus hypoplasia in allergic chronic rhinosinusitis patients compared to those without allergy [4]. Natsis et al. showed that anatomical variations characterized by the unusual spread of frontal

Table $\mathbf{2}$ Comparison of the right and the left sides in terms of the frequency of frontal sinus types

\begin{tabular}{|c|c|c|c|c|c|c|c|c|}
\hline & \multicolumn{8}{|c|}{ Right frontal sinus type } \\
\hline & \multicolumn{2}{|c|}{ Small } & \multicolumn{2}{|c|}{ Medium-sized } & \multicolumn{2}{|c|}{ Large } & \multicolumn{2}{|c|}{ Total } \\
\hline & $N$ & $\%$ & $N$ & $\%$ & $N$ & $\%$ & $N$ & $\%$ \\
\hline \multicolumn{9}{|c|}{ Left frontal sinus type } \\
\hline Small & 72 & 74.2 & 15 & 5.6 & 1 & 2.6 & 88 & 21.8 \\
\hline Medium-sized & 24 & 24.7 & 231 & 86.2 & 9 & 23.1 & 264 & 65.3 \\
\hline Large & 1 & 1.0 & 22 & 8.2 & 29 & 74.4 & 52 & 12.9 \\
\hline Total & 97 & 100.0 & 268 & 100.0 & 39 & 100.0 & 404 & 100.0 \\
\hline
\end{tabular}

Table 4 Relationship between the frequency of frontal sinusitis and age and gender

\begin{tabular}{llll}
\hline & Frontal sinusitis & $P$ \\
\cline { 2 - 3 } & Positive $(\boldsymbol{N = 1 5 6 )}$ & Negative $(\boldsymbol{N = 2 4 8 )}$ & \\
\hline Age (mean \pm SD) & $40.98 \pm 14.59$ & $39.05 \pm 15.86$ & 0.151 \\
Gender [N (\%)] & & & \\
$\quad$ Female & $60(38.5)$ & $129(52.0)$ & $0.008^{*}$ \\
$\quad$ Male & $96(61.5)$ & $119(48.0)$ & \\
\hline
\end{tabular}

*Significant at 0.05 level; Mann-Whitney $U$ test for numerical data, chi-square test for categorical data 
Table 5 Relationship between the type of frontal sinus and the frequency of sinusitis for both sides

\begin{tabular}{lllll}
\hline & Small & Medium-sized & Large & \multicolumn{1}{c}{} \\
\hline Right frontal sinusitis $(\boldsymbol{N = 1 2 0})$ & $14 / 97(14.4 \%)$ & $92 / 268(34.3 \%)$ & $14 / 39(35.9 \%)$ & $0.001^{*}$ \\
Left frontal sinusitis $(\boldsymbol{N = 1 2 8 )}$ & $14 / 88(15.9 \%)$ & $88 / 264(33.3 \%)$ & $26 / 52(50.0 \%)$ & $0.001^{*}$ \\
$\boldsymbol{P}$ & 0.843 & 0.154 & 0.066 \\
\hline
\end{tabular}

*Significant at 0.05 level; chi-square test. Bonferroni test was used in further comparison of the ratios following chi-square test

sinuses above the orbital roof may support the development of orbital complications, and they suggested that such cases should be considered as "high-risk" cases in terms of orbital complications during the course of frontal sinusitis [5]. Vazquez et al. emphasized that the incidence of both the orbital and intracranial complications of frontal sinusitis is greatly influenced by the anatomy and size of the frontal sinuses [3].

Although the anatomical variations of the frontal sinus are closely related to the development and course of a disease that is very common, such as sinusitis, it is surprising that a common language has not yet been developed in describing the anatomical structure of the frontal sinus. Previous attempts to define the frontal sinus types were based solely on the dimensions of the sinus $[9,10]$. However, since the orbital contact area of the frontal sinus plays a critical role in the clinical course of the disease, the classification proposed by Stokovic et al. appears to be more functional than a dimension-based approach. In our retrospective review, we recorded the findings of frontal sinusitis in $38.6 \%$ of the study group. And, in accordance with the previous literature, we did not note side- or age-related differences in the frequency of frontal sinusitis [20,21]. Using the classification proposed by Stokovic et al., we found a significant relationship between the type of the frontal sinus and the frequency of frontal sinusitis. We showed that the prevalence of sinusitis in medium-sized and large sinuses is significantly higher than that in small sinuses. We believe that this original finding we obtained in our study will contribute to the diagnostic evaluation of sinusitis in clinical practice.

The major limitation of our study is that we made only an imaging-based evaluation of frontal sinusitis. However, testing the relation between the anatomic type and the presence of the disease based on a comprehensive clinico-radiological evaluation would be a more accurate assessment. Our second limitation is that we did not include the evaluation of the complications of frontal sinusitis. Third, pathologies involving the frontal sinuses other than infection, such as malignancies, have not been evaluated. Further comprehensive studies adopting a clinico-radiological approach are needed to reach accurate and inclusive comments regarding the clinical relevance and usefulness of this newly-introduced anatomical classification.

\section{Conclusions}

We typed frontal sinuses according to the classification proposed by Stokovic et al. [15] and showed a significant relationship between frontal sinus type and frequency of frontal sinusitis. The result obtained in this study may be considered the first step in demonstrating the clinical benefit of this classification. However, there is no doubt that further comprehensive studies with large series are needed to fully determine the clinical relevance of this newly introduced classification system.

\section{Abbreviations \\ IFAC: International Frontal Sinus Anatomy Classification; CT: Computed tomography}

\section{Acknowledgements}

Not applicable.

\section{Authors' contributions}

MÖ: Project development, data collection, manuscript writing. RPK: Data collection and manuscript writing. BÖ and $\mathrm{H}$ : Data collection. All authors have read and approved the manuscript.

\section{Funding}

This research did not receive any specific grant from funding agencies in the public, commercial, or not-for-profit sectors.

\section{Availability of data and materials}

The datasets used and/or analyzed during the current study are available from the corresponding author on reasonable request.

\section{Declarations}

Ethics approval and consent to participate

Approval was provided by University of Health Sciences, Dışkapı Yıldırım Beyazıt Training and Research Hospital (Reference No: 64/04). Consent to participate is not applicable as it is a retrospective study.

\section{Consent for publication}

The need for consent for publication was waived due to the retrospective design of the study.

\section{Competing interests}

The authors declare that they have no conflict of interest.

\section{Author details}

'Department of Radiology, University of Health Sciences, Dışkapı Yıldırım Beyazıt Training and Research Hospital, Ankara, Turkey. ${ }^{2}$ Department of Otorhinolaryngology, University of Health Sciences, Dışkapı Yıldııım Beyazıt Training and Research Hospital, Ankara, Turkey. ${ }^{3}$ Department of Anatomy, Faculty of Dentistry, Yıldırım Beyazıt University, Ankara, Turkey. 
Received: 10 March 2021 Accepted: 5 April 2021

Published online: 21 April 2021

\section{References}

1. Danesh-Sani SA, Bavandi R, Esmaili M (2011) Frontal sinus agenesis using computed tomography. J Craniofac Surg 22(6):e48-e51. https://doi.org/10.1 097/SCS.0b013e318231e26c

2. Harris AM, Wood RE, Nortjé CJ, Thomas CJ (1987) The frontal sinus: forensic fingerprint? A pilot study. J Forensic Odontostomatol 5(1):9-15

3. Vazquez A, Baredes S, Setzen M, Eloy JA (2016) Overview of frontal sinus pathology and management. Otolaryngol Clin North Am 49(4):899-910. https://doi.org/10.1016/j.otc.2016.03.014

4. Tezer MS, Tahamiler R, Canakcioglu S (2006) Computed tomography findings in chronic rhinosinusitis patients with and without allergy. Asian Pac J Allergy Immunol 24(2-3):123-127

5. Natsis K, Karabatakis V, Tsikaras P, Chatzibalis T, Stangos A, Stangos N (2004) Frontal sinus anatomical variations with potential consequences for the orbit. Study on cadavers. Morphologie 88(280):35-38. https://doi.org/10.101 6/S1286-0115(04)97997-0

6. Johari HH, Mohamad I, Sachlin IS, Aziz ME, Mey TY, Ramli RR (2018) A computed tomographic analysis of frontal recess cells in association with the development of frontal sinusitis. Auris Nasus Larynx 45(6):1183-1190. https://doi.org/10.1016/j.anl.2018.04.010

7. DelGaudio JM, Hudgins PA, Venkatraman G, Beningfield A (2005) Multiplanar computed tomography analysis of frontal recess cells: effect on frontal isthmus size and frontal sinusitis. Arch Otolaryngol Head Neck Surg 131(3):230-235. https://doi.org/10.1001/archotol.131.3.230

8. Angélico FV Jr, Rapoport PB (2013) Analysis of the agger nasi cell and frontal sinus ostium sizes using computed tomography of the paranasal sinuses. Braz J Otorhinolaryngol 79(3):285-292. https://doi.org/10.5935/18088694.20130052

9. Guerram A, Le Minor JM, Renger S, Bierry G (2014) Brief communication: the size of the human frontal sinuses in adults presenting complete persistence of the metopic suture. Am J Phys Anthropol 154(4):621-627. https://doi. org/10.1002/ajpa.22532

10. Yuksel Aslier NG, Karabay N, Zeybek G, Keskinoglu P, Kiray A, Sutay S, Ecevit MC (2016) The classification of frontal sinus pneumatization patterns by CTbased volumetry. Surg Radiol Anat 38(8):923-930. https://doi.org/10.1007/ s00276-016-1644-7

11. Bent J, Kuhn FA, Cuilty C (1994) The frontal cell in frontal recess obstruction. Am J Rhinol 8(4):185-191. https://doi.org/10.2500/105065894781874278

12. Wormald PJ, Hoseman W, Callejas C, Weber RK, Kennedy DW, Citardi MJ, Senior BA, Smith TL, Hwang PH, Orlandi RR, Kaschke O, Siow JK, Szczygielski K, Goessler U, Khan M, Bernal-Sprekelsen M, Kuehnel T, Psaltis A (2016) The International Frontal Sinus Anatomy Classification (IFAC) and classification of the Extent of Endoscopic Frontal Sinus Surgery (EFSS). Int Forum Allergy Rhinol 6(7):677-696. https://doi.org/10.1002/alr.21738

13. Sharma SA (2017) Frontal sinus outflow tract: multi-detector $C T$ assessment. Egypt J Radiol Nucl Med 48(4):897-903. https://doi.org/10.1016/j.ejrnm.2017. 06.012

14. Gotlib T, Kołodziejczyk P, Kuźmińska M, Bobecka-Wesołowska K, Niemczyk K (2019) Three-dimensional computed tomography analysis of frontoethmoidal cells: a critical evaluation of the International Frontal Sinus Anatomy Classification (IFAC). Clin Otolaryngol 44(6):954-960. https://doi. org/10.1111/coa.13412

15. Štoković N, Trkulja V, Čuković-Bagić I, Lauc T, Grgurević L (2018) Anatomical variations of the frontal sinus and its relationship with the orbital cavity. Clin Anat 31(4):576-582. https://doi.org/10.1002/ca.22999

16. Ah-See KW, Evans AS (2007) Sinusitis and its management. BMJ 334(7589): 358-361. https://doi.org/10.1136/bmj.39092.679722.BE

17. Fokkens $W$, Lund $V$, Bachert $C$, Clement $P$, Helllings $P$, Holmstrom $M$, Jones N, Kalogjera L, Kennedy D, Kowalski M, Malmberg H, Mullol J, Passali D, Stammberger H, Stierna P, EAACI (2005) EAACI position paper on rhinosinusitis and nasal polyps executive summary. Allergy 60(5):583-601. https://doi.org/10.1111/j.1398-9995.2005.00830.x

18. Betz Ch S, Issing W, Matschke J, Kremer A, Uhl E, Leunig A (2008) Complications of acute frontal sinusitis: a retrospective study. Eur Arch Otorhinolaryngol 265(1):63-72

19. Goldberg AN, Oroszlan G, Anderson TD (2001) Complications of frontal sinusitis and their management. Otolaryngol Clin North Am 34(1):211-225. https://doi.org/10.1016/S0030-6665(05)70307-8
20. Tarp B, Fiirgaard B, Christensen T, Jensen JJ, Black FT (2000) The prevalence and significance of incidental paranasal sinus abnormalities on MRI. Rhinology 38(1):33-38

21. Nazri M, Bux SI, Tengku-Kamalden TF, Ng KW, Sun Z (2013) Incidental detection of sinus mucosal abnormalities on $C T$ and MRI imaging of the head. Quant Imaging Med Surg 3(2):82-88. https://doi.org/10.3978/j. issn.2223-4292.2013.03.06

\section{Publisher's Note}

Springer Nature remains neutral with regard to jurisdictional claims in published maps and institutional affiliations.

\section{Submit your manuscript to a SpringerOpen ${ }^{\circ}$ journal and benefit from:}

- Convenient online submission

Rigorous peer review

- Open access: articles freely available online

- High visibility within the field

- Retaining the copyright to your article

Submit your next manuscript at $\boldsymbol{\nabla}$ springeropen.com 